\title{
ESTUDO SOBRE A INFLUENNCIA DA UMIDADE NA TERMOFIXAÇÃO DE ESTAMPAS COM CORANTES REATIVOS EM TECIDOS DE ALGODÃO
}

\author{
R. N. BEZERRA ${ }^{1}$, J. M. ROSA ${ }^{1,2}$, A. M. F. FILETI², E. B. TAMBOURGI², J. C. C. \\ SANTANA $^{3}$ \\ ${ }^{1}$ Faculdade de Tecnologia SENAI Antoine Skaf - Pós-Graduação em Química Têxtil \\ ${ }^{2}$ UNICAMP - Faculdade de Engenharia Química \\ ${ }^{3}$ UNINOVE - Programa de Mestrado em Engenharia de Produção
}

\begin{abstract}
RESUMO - Este estudo comparou dois processos de termofixação de estampas com os corantes RY84, RR120 e RB160. Foram avaliados os itens diferença de intensidade colorística $\left(K \cdot \mathrm{S}^{-1}\right)$, desvio total entre as cores $\left(\Delta \mathrm{E}^{*}\right)$, diferença de energia térmica entre os processos e solidez das cores à água. O processo em presença de umidade demonstrou maior rendimento, ou seja, para que os $\mathrm{K} \cdot \mathrm{S}^{-1}$ ficassem iguais nos dois processos, seria necessário um aumento de 25\% na concentração de RY84, $8 \%$ na concentração de RR120 e 13\% na concentração de RB160 no processo de termofixação sem umidade. Os desvios apresentados foram de 5,54 para o RY84, 1,47 para o RR120 e 4,91 para o RB160, sendo que valores abaixo de 1,5 são aceitáveis dentro da indústria de confecções. O processo em presença de umidade despende maior quantidade de energia térmica, $6,83 \cdot 10^{3} \mathrm{~J}$, consumindo $2,00 \cdot 10^{-4} \mathrm{~m}^{3}$ a mais de gás combustível e gerando emissão de $4,32 \cdot 10^{-10} \mathrm{~kg}$ de $\mathrm{CO}_{2}$. Os testes de solidez não apresentaram diferença significativa.
\end{abstract}

\section{INTRODUÇÃO}

A estamparia têxtil, uma arte milenar, é um processo de coloração onde somente um lado do tecido é colorido. Pode ser definido como sendo um conjunto de figuras ou desenhos impressos nos tecidos formando uma padronagem (ANDREONI, 2008). As estampas podem ser efetuadas com pigmentos ou com corantes, sendo a mais comum efetuada com pigmentos. Cerca de $20 \%$ de todos os produtos têxteis são estampados e destes, de $45 \%$ a $50 \%$ fazem uso de pigmentos, o método mais simples e econômico. Porém, as estampas com pigmento proporcionam um toque mais áspero do que as estampas produzidas com corantes (ANDREONI, 2008; MALUF e KOLBE, 2003). Vem sendo realizada e orientada pela "experiência" que, em grande parte, tem substituído a investigação de parâmetros que permitam controlar com exatidão tais processos (GIORDANO, 2002; ANDREAUS et al., 2010). Segundo Prado (2013), em 2012 os tecidos compostos por fibras celulósicas foram os mais produzidos, quando comparados em relação às demais fibras no mesmo período. Este fato que levou a opção por trabalhar com tecido produzido com fibras de algodão, em estampas produzidas com corantes reativos, corantes estes os mais consumidos atualmente no Brasil (ABIQUIM, 2013). 


\subsection{Corantes Reativos}

Os corantes reativos ainda dominam a estamparia com corantes em tecidos de fibras celulósicas principalmente pela facilidade na produção. As pastas de estampar com corantes reativos em geral se conservam bem em ambientes frios por até uma semana. Esses corantes consistem em uma das grandes classes de corantes utilizadas para estamparia em algodão e outros materiais celulósicos. Reagem quimicamente com a celulose para formar uma ligação química covalente e proporcionam altos índices de solidezes à fricção e aos tratamentos úmidos (KONS, 2010; PEARSTINE, 2006; YAMANE, 2008). Ha dois grandes grupos de corantes reativos, os Vinilsulfônicos (VS) que reagem por adição nucleofílica e os Halogenoheterocíclicos (HHC), grupo do qual fazem parte os corantes do tipo monoclorotriazina (MCT), que reagem por substituição nucleofílica e que foram abordados neste estudo.

\section{MATERIAIS E MÉTODOS}

\subsection{Materiais}

Equipamentos: Espectrofotômetro Konica-Minolta CM-3600d, Termofixador-Vaporizador Mathis GD-B; Mesa de estampar de acionamento eletromagnético Mathis SILK, Misturador Mathis M, Viscosímetro Brookfield Analógico Modelo LVT, Perspirômetro, Esticador pneumático Screen Printing HB, Tensiômetro HB, Lavadora Suzuki com velocidade periférica de $40 \mathrm{~m} \cdot \mathrm{min}^{-1}$, três aletas com $5 \mathrm{~cm}$ de altura e tempo de ciclo igual a $17 \mathrm{~s}$ e Gerador de Vapor Etna GHV-2000 com capacidade de produção igual a 5,56 $10^{-1} \mathrm{~kg} \cdot \mathrm{s}^{-1}$, Pressão Máxima de Trabalho Admissível (PMTA) igual a 1,0 MPa operando com eficiência de 85\%, este último utilizado como parâmetro para a modelagem da termodinâmica.

Reagentes: Corantes RB 160, RR 120 e RY 84 (Basf), alginato de sódio 98\%, etanol 95\%, uréia 90\%, carbonato de sódio 95\% (Manchester) e anti-redutor (Clariant) para as pastas de estampar; adesivo sintético de secagem rápida para preparação da matriz; Ácido Acético 95\% (Manchester), Retardante (Basf) e Sequestrante (Goldenquímica) para a lavagem posterior.

Materiais diversos: quadro de alumínio, tecido plano de poliéster multifilamento de densidade linear igual a 90 fios $\mathrm{cm}^{-1}$ para a preparação do quadro; malha alvejada (ROSA, 2012) de algodão 100\%, fio 30/1 Ne cardado, 16 colunas/cm e 18 malhas/cm, com largura tubular de 92 $\mathrm{cm}$ e gramatura igual a 130 gramas por $\mathrm{cm}^{2}$, produzida em equipamento circular monofrontura Orizio, John/C model, 120 alimentadores, diâmetro de 30 polegadas e velocidade de 30 RPM, tecido utilizado para a estampa.

\subsection{Metodologia}

Colocou-se o tecido de poliéster sobre o quadro de alumínio e aplicou-se a tensão através das garras (Esticador HB), efetuando-se em seguida a leitura da tensão obtida (Tensiômetro HB), de valor igual a $38 \mathrm{~N} \cdot \mathrm{cm}^{-1}$; colou-se a tela de poliéster no quadro de alumínio; efetuou-se a lavagem da matriz com água e detergente não iônico; secou-se a matriz durante 30 minutos a 50 ${ }^{\circ} \mathrm{C}$; para o preparo das pastas coloridas, utilizou-se as quantidades de produtos descritas na Tabela 1, mantendo a mistura sob agitação constante de 1300 RPM durante 10 minutos (Misturador Mathis M); efetuou-se a medida da viscosidade das pastas (Viscosímetro Brookfield, spindler 6, 10 RPM), cujos resultados encontram-se descritos na Tabela 1. 
Tabela 1 - Receitas das pastas coloridas (SENAI, 2012).

\begin{tabular}{|c|c|c|c|c|}
\hline \multirow{2}{*}{ Produtos } & \multicolumn{3}{|c|}{ Corante } & \multirow{2}{*}{ Viscosidade (cP) } \\
\hline & RY84 & RR120 & RB160 & \\
\hline Alginato a $4 \%$ & 500 & 500 & 500 & \multirow{9}{*}{$\begin{array}{c}4000 \text { à } \\
26^{\circ} \mathrm{C}\end{array}$} \\
\hline RY 84 & 30 & - & - & \\
\hline RR 120 & - & 30 & - & \\
\hline RB 160 & - & - & 30 & \\
\hline Uréia & 100 & 100 & 100 & \\
\hline Anti-redutor & 10 & 10 & 10 & \\
\hline Carbonato de Sódio 1:3 & 90 & 90 & 90 & \\
\hline Água & 270 & 270 & 270 & \\
\hline TOTAL & 1000 & 1000 & 1000 & \\
\hline
\end{tabular}

Colocou-se o tecido na mesa (Mesa de estampar Mathis SILK), adicionou-se a pasta na matriz e, em seguida, com intensidade eletromagnética em nível 6, vareta de diâmetro igual a 10 $\mathrm{mm}$ e velocidade igual a $3 \mathrm{~m} \cdot \mathrm{min}^{-1}$, executou-se somente uma passagem.

Após o processo de estampa, as amostras foram divididas em duas partes, sendo uma delas submetida em condições de termofixação em presença de umidade (vaporização com $280 \mathrm{~g}$ de água por $\mathrm{m}^{3}$ ) e a outra em condições de termofixação sem presença de umidade, ambas em temperatura de $105^{\circ} \mathrm{C}$ durante 10 minutos, com velocidade do motor de circulação de ar em 1000 RPM (Termofixador-Vaporizador Mathis GD-B).

Todas as amostras estampadas foram submetidas em lavagem posterior (Lavadora Suzuki), em relação de banho igual a 1:15 (15 litros de água para cada $1 \mathrm{~kg}$ de substrato lavado), utilizando-se $0,5 \mathrm{~g} \cdot \mathrm{L}^{-1}$ de retardante $(\mathrm{A}), 0,5 \mathrm{~mL} \cdot \mathrm{L}^{-1}$ de ácido acético (B) e $0,5 \mathrm{~g} \cdot \mathrm{L}^{-1}$ de sequestrante (C). Todas as etapas efetuadas no processo de lavagem estão representadas graficamente na Figura 1.

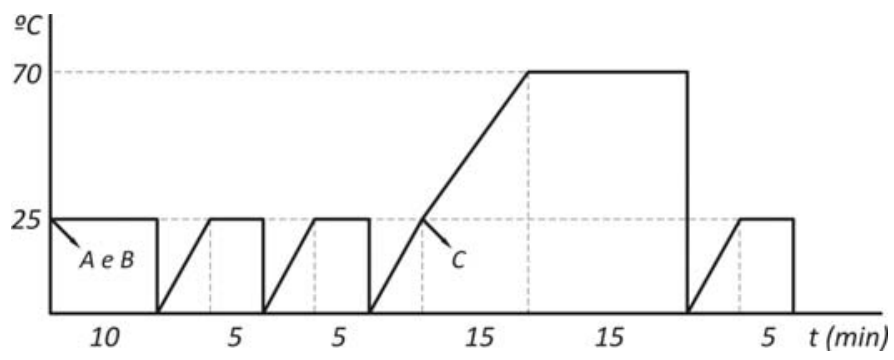

Figura 1 - Etapas do processo de lavagem posterior a termofixação (SENAI, 2012).

Para comparação do rendimento entre os processos, avaliou-se reflectância (R) das cores através de espectrofotometria visível, sob iluminante D65 10², Sistema CIELab (CM-3600d), calculando-se a intensidade colorística $\left(\mathrm{K} \cdot \mathrm{S}^{-1}\right)$ através da Equação 1, de Kubelka-Munk (SILVA et al., 2012).

$$
K \cdot S^{-1}=(1-R)^{2} \cdot(2 R)^{-1}
$$


Determinou-se, também por espectrofotometria visível, os valores de desvios $\Delta \mathrm{L}^{*}, \Delta \mathrm{a}^{*}$ e $\Delta \mathrm{b}^{*}$, calculando-se o Desvio Total ( $\Delta \mathrm{E}^{*}$ ) através da Equação 2 (SILVA et al., 2012), a seguir.

$$
\Delta E^{*}=\left[\left(\Delta L^{*}\right)^{2}+\left(\Delta a^{*}\right)^{2}+\left(\Delta b^{*}\right)^{2}\right]^{1 / 2}
$$

Para determinação da correlação $[\mathrm{R}] / \mathrm{K} \cdot \mathrm{S}^{-1}$, foram desenvolvidas curvas de calibração com valores de $[\mathrm{R}]$ descritos na Tabela 2 , juntamente com os respectivos valores de $\mathrm{K} \cdot \mathrm{S}^{-1}$ obtidos. Através destes dados foi obtido os Gráfico 1, juntamente com as equações e os valores de regressão linear. Esta correlação é necessária para calcular a diferença em " $\mathrm{g} \cdot \mathrm{kg}^{-1}$ ” de corante entre os ensaios com e sem presença de umidade, já que o $\mathrm{K} \cdot \mathrm{S}^{-1}$ é adimensional, demonstrando somente a intensidade colorística.

Tabela 2 - Valores de concentração e K·S ${ }^{-1}$ para os corantes RY84, RR120 e RB160.

\begin{tabular}{cccc}
\hline $\mathrm{g} \cdot \mathrm{kg}^{-1}$ & \multicolumn{3}{c}{$\mathrm{K} \cdot \mathrm{S}^{-1}$} \\
& $\mathrm{RY84}(\mathrm{em} \mathrm{430} \mathrm{nm})$ & $\mathrm{RR} 120(\mathrm{em} 550 \mathrm{~nm})$ & $\mathrm{RB} 160(\mathrm{em} 620 \mathrm{~nm})$ \\
\hline 15,00 & 10,183 & 13,942 & 13,723 \\
10,00 & 7,4051 & 9,0651 & 9,1462 \\
7,50 & 6,2497 & 6,7484 & 7,8467 \\
6,00 & 5,0156 & 5,7576 & 5,6778 \\
5,00 & 4,2084 & 4,5281 & 5,4411 \\
\hline
\end{tabular}

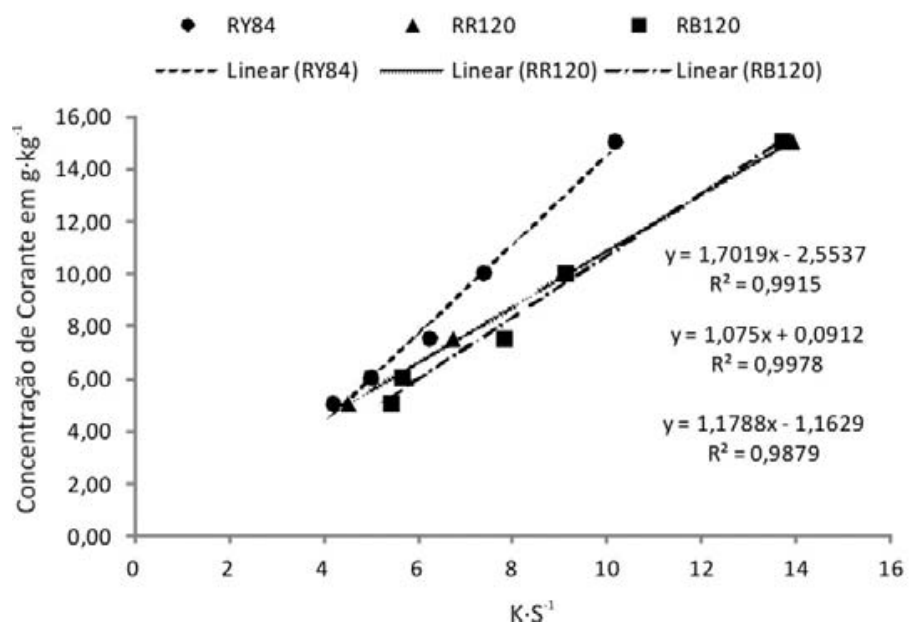

Gráfico 1 - Correlações [Corantes] $/ \mathrm{K} \cdot \mathrm{S}^{-1}$.

O ensaio de solidez a água foi executado segundo norma NBR ISO 105-E01:2011. Antes dos ensaios, as amostras foram condicionadas por $24 \mathrm{~h}$ em atmosfera padrão de temperatura igual a $20 \pm 2{ }^{\circ} \mathrm{C}$ e umidade relativa igual a $65 \pm 2 \%$ (GUN e TIBER, 2011). Os resultados foram avaliados por espectrofotometria Vis, sob iluminante D65, $10^{\circ}$. 


\section{RESULTADOS}

\subsection{Avaliação das cores}

Os valores de intensidade colorística $\left(\mathrm{K} \cdot \mathrm{S}^{-1}\right)$, desvios parciais $\left(\Delta \mathrm{a}^{*}, \Delta \mathrm{b}^{*}\right.$ e $\left.\Delta \mathrm{L}^{*}\right)$ e desvio total $\left(\Delta \mathrm{E}^{*}\right)$ estão descritos na Tabela 3. Estes valores foram mensurados tendo como "ponto zero" a leitura da amostra alvejada, entretanto, o delta foi calculado entre as cores obtidas.

Tabela 3 - Desvios entre as amostras com e sem presença de umidade.

\begin{tabular}{|c|c|c|c|c|}
\hline Umidade & Parâmetros & RY84 & RR120 & RB160 \\
\hline \multirow{4}{*}{ COM } & $\mathrm{K} \cdot \mathrm{S}^{-1}$ & 18,09 & 19,25 & 20,65 \\
\hline & $\mathrm{L}^{*}$ & 58,88 & 39,05 & 22,56 \\
\hline & $a^{*}$ & 39,89 & 58,54 & $-0,78$ \\
\hline & $b^{*}$ & 62,37 & 3,92 & $-16,36$ \\
\hline \multirow{4}{*}{ SEM } & $\mathrm{K} \cdot \mathrm{S}^{-1}$ & 13,64 & 16,75 & 17,27 \\
\hline & $\mathrm{L}^{*}$ & 56,82 & 40,24 & 24,70 \\
\hline & $a^{*}$ & 37,15 & 58,19 & $-4,53$ \\
\hline & $\mathrm{b}^{*}$ & 58,02 & 3,14 & $-18,70$ \\
\hline \multicolumn{2}{|c|}{$\Delta \mathrm{K} \cdot \mathrm{S}^{-1}$} & 4,45 & 2,50 & 3,38 \\
\hline \multicolumn{2}{|c|}{$\Delta \mathrm{L}^{*}$} & 2,06 & $-1,19$ & $-2,14$ \\
\hline \multicolumn{2}{|c|}{$\Delta \mathrm{a}^{*}$} & 2,74 & 0,35 & 3,75 \\
\hline \multicolumn{2}{|c|}{$\Delta \mathrm{b}^{*}$} & 4,35 & 0,78 & 2,34 \\
\hline \multicolumn{2}{|c|}{$\Delta \mathrm{E}^{*}$} & 5,54 & 1,47 & 4,91 \\
\hline
\end{tabular}

As diferenças entre os valores de $\mathrm{K} \cdot \mathrm{S}^{-1}$ ficaram em 4,45 para o RY84; 2,50 para o RR120 e 3,38 para o RB160. Observou-se que há um comportamento particular para cada corante, isto significa que pode haver diferenças de tonalidade quando utilizados em tricromias.

De acordo com as equações de correlação [Corante] $/ \mathrm{K} \cdot \mathrm{S}^{-1}$, calculou-se essa diferença em $\mathrm{g} \cdot \mathrm{kg}^{-1}$, onde obteve-se o valor de 7,59 para o RY84; 2,94 para o RR120 e 4,01 para o RB160. Este fato demonstra que, para obter-se a mesma intensidade em cores termofixadas com e sem presença de umidade utilizando-se estes corantes, seria necessário acrescentar esta diferença nos processos com termofixação sem presença de umidade. Quanto aos valores de $\Delta \mathrm{E}^{*}$, ficaram acima de 2,0 o RY84 e o RB160, valores de $\Delta \mathrm{E}^{*}$ abaixo de 1,5 são aceitáveis dentro do mercado.

\subsection{Solidez à agua}

As diferenças entre os valores dos índices de solidez à água não foram significativas. As notas obtidas por avaliação espectrofotométrica estão dispostas na Tabela 4, onde se pode observar a semelhança entre os valores para os dois processos. 
Tabela 4 - Notas relativas ao ensaio de solidez à água.

\begin{tabular}{ccccccc}
\hline \multirow{2}{*}{ Parâmetros } & \multicolumn{2}{c}{ RY84 } & \multicolumn{2}{c}{ RR120 } & \multicolumn{2}{c}{ RB160 } \\
& A & T & A & T & A & T \\
\hline Com UMIDADE & 5 & $4 / 5$ & 5 & 5 & 5 & $4 / 5$ \\
Sem UMIDADE & 5 & $4 / 5$ & 5 & 5 & 5 & 5 \\
\hline
\end{tabular}

Legenda: $A$ = Alteração $T=$ Transferência

\subsection{Termodinâmica}

Segundo Atkins e Jones (2011), para vaporizar um mol de água na forma líquida (18,02 g em $25^{\circ} \mathrm{C}$ ), deve-se fornecer $44,0 \mathrm{~kJ}$ de energia na forma de calor. Para o processo de termofixação em presença de umidade foram necessários $280 \mathrm{~g}$ de vapor de água por metro cúbico. Para esse montante, o consumo extra de energia em relação ao processo de termofixação sem presença de umidade seria de $683,68 \mathrm{~kJ}$ ou $6,83 \cdot 10^{3} \mathrm{~J}$.

De acordo com o fornecedor de gás combustível, a Companhia de Gás de São Paulo (Comgás), o gás comercializado e utilizado no gerador de vapor para este estudo possui 89,0\% de metano, 6,0\% de etano e 1,8\% de propano. Baseando-se no Poder Calorífico Inferior (PCI) descrito pela norma ASTM D 3588-98 (JOSÉ, 2004), como sendo 3,70 $10^{7} \mathrm{~J} \cdot \mathrm{m}^{-3}$ para o metano, $7,00 \cdot 10^{7} \mathrm{~J} \cdot \mathrm{m}^{-3}$ para o etano e $9,23 \cdot 10^{7} \mathrm{~J} \cdot \mathrm{m}^{-3}$ de propano, o PCI calculado de gás combustível foi de $4,02 \cdot 107 \mathrm{~J} \cdot \mathrm{m}^{-3}$.

Nestas condições, para calcular o volume de gás combustível necessário para a obtenção de $6,83 \cdot 10^{3} \mathrm{~J}$, utilizou-se a Equação (3).

$$
V=\frac{Q}{4,02 \cdot 10^{7} \cdot E_{G V}}
$$

Onde: $V$ = volume de gás combustível $\left(\mathrm{m}^{3}\right) ; \mathrm{Q}=$ energia despendida $(J) ; E_{G V}=$ eficiência do gerador de vapor (\%).

Adotando-se um gás ideal nas condições normais de pressão e temperatura e aplicando-se a Equação 3, determinou-se que o consumo de gás combustível seria de $2,00 \cdot 10^{-4} \mathrm{~m}^{3}$, o que geraria $4,32 \cdot 10^{-10} \mathrm{~kg}$ de $\mathrm{CO}_{2}$, quantidade obtida através da Equação (4).

$$
m_{\mathrm{CO}_{2}}=\frac{P \cdot V \cdot 4,40 \cdot 10^{-2} \cdot F_{C}}{R \cdot T}
$$

Onde: $\mathrm{mCO}_{2}=\mathrm{kg} ; \mathrm{P}=101,3 \mathrm{kPa} ; \mathrm{V}=\mathrm{m}^{3}(\mathrm{Eq} .3) ; R=8314 \mathrm{~m}^{3} \cdot \mathrm{kPa}^{\mathrm{mol}} \mathrm{mol}^{-1} \cdot \mathrm{K}^{-1} ; \mathrm{T}=273,15 \mathrm{Ke}$ FC $=$ fator de carbono na mistura, igual a 1,10 . 


\section{CONCLUSÃO}

Através do estudo com estes corantes, demonstrou-se que há um rendimento maior quando há presença de umidade na termofixação, entretanto, este rendimento é diferente para cada corante. As quantidades de corantes utilizadas para ambos os processos foram de $30 \mathrm{~g} \cdot \mathrm{kg}^{-1}$, mas para que se obtenha rendimento colorístico semelhante, é necessário um aumento na quantidade de RY84 para 37,59 $\mathrm{g} \cdot \mathrm{kg}^{-1}$ (25\%), 32,49 $\mathrm{g} \cdot \mathrm{kg}^{-1}$ (8\%) para a quantidade de RR120 e 34,01 $\mathrm{g} \cdot \mathrm{kg}^{-1}$ (13\%) na quantidade de RB160, todas elas para receitas com posterior termofixação sem presença de umidade. Este fato demonstra que os corantes possuem comportamento diferente e isto pode acarretar diferentes tonalidades em cores desenvolvidas com esta tricromia.

Há também a diferença no consumo de energia. O processo de termofixação com presença de umidade gera um consumo de $6,83 \cdot 10^{3} \mathrm{~J}$ a mais do que o processo sem umidade, consumindo $2,00 \cdot 10^{-4} \mathrm{~m}^{3}$ a mais de gás combustível, gerando emissão de $4,32 \cdot 10^{-10} \mathrm{~kg}$ de $\mathrm{CO}_{2}$.

Importante acrescentar que o estudo restringiu-se apenas na influência da umidade em três corantes de classe específica. Além de outros corantes utilizados no mercado, como os vinilsulfônicos ou os bi-heterofuncionais, há também outros fatores que podem influenciar na termofixação de corantes reativos, como a temperatura ou ainda a quantidade de álcali.

\section{Agradecimentos}

Os autores agradecem ao Diretor da Faculdade de Tecnologia SENAI Antoine Skaf, Prof. Marcelo Costa, pela disponibilização das dependências da escola para a execução dos ensaios e ao Prof. Marco Aurélio Rodrigues, pelas ideias e apoio técnico. 


\section{REFERÊNCIAS}

ABIQUIM: Associação Brasileira da Indústria Química. Disponível e acessado em 02/11/2013, www.abiquim.org.br/comissao/setorial/corantes-pigmentos/especificidade/historico-aplicacao, 2013.

ANDREAUS, J.; DALMOLIN, M.C.; OLIVEIRA JUNIOR, I.B.; BARCELLOS, I.O.: Aplicação de ciclodextrinas em processos têxteis. Quim. Nova, vol.33, n.4, p.929-937, 2010.

XYZTR, S.; ABCDEF, M.; GHIJ, D. Título completo do artigo. Chem. Eng. J., v. 10, p. 10-20, 2009.

ANDREONI, M.A.L.: Estamparia Têxtil: Uma estratégia na diferenciação do produto da manufatura do vestuário de moda, Dissertação, Engenharia de Produção, Universidade Paulista, São Paulo, 2008.

ATKINS, P.W.; JONES, J.: "Termodinâmica: A Primeira Lei". In: Princípios de Química, 5a edição, Editora Bookman, p.320, 2011.

GIORDANO, J.B.: Caracterização Físico-Química da resina acrílica aplicada na estamparia têxtil, Dissertação, UNICAMP, 2002.

GUN, A.D.; TIBER, B.: Color, colorfastness and abrasion properties of 50/50 bamboo/cotton blended plain knitted fabrics in three different stitch lengths. Text. Res. J., 81: 1903-1915, 2011.

JOSÉ, H.J., 2004. Combustão e Combustíveis. Departamento de Engenharia Química e Alimentos. Universidade Federal de Santa Catarina - UFSC, 2004.

KONS, T.: Acompanhamento e análise crítica dos processos para identificação das causas dos problemas encontrados em estamparia. Trabalho de Conclusão do Curso de Bacharel em Química, Universidade Regional de Blumenal, Blumenau, 2010.

MALUF, E.; KOLBE, W.: Dados técnicos para a indústria têxtil, 2. Ed., São Paulo: IPT, 2003.

PEARSTINE, Kathryn. INK. Development manager. Versão para português pela Leila e J. C. Macedo. Siderquímica do Brasil, 2006.

PRADO M.V.: Relatório Setorial da Indústria Têxtil Brasileira, ABIT/IEMI, 2013.

ROSA, J.M.; TAMBOURGI, E.B.; SANTANA, J.C.C.; CARMO, R.A.S,: Estudo de variáveis e de custos ecológicos no pré-tratamento de tecidos de algodão com peróxido de hidrogênio. In: Quim. Têxtil, n.109, p. 30-38, 2012.

SENAI - Escola SENAI "Francisco Matarazzo”: Processos Produtivos Têxteis III, 2012.

SILVA, B.B.; BEZERRA, R.N.; ROSA, J.M.; TAMBOURGI, E.B.; SANTANA, J.C.C.: Influência da estiragem mecânica nas propriedades físico-químicas de multifilamentos de poliamida 6. In: Quim. Têxtil, n.112, p. 30-38, 2012.

YAMANE, L.A.: Estamparia Têxtil, Dissertação, ECA-USP, 2008.

www.worlddyevariety.com/reactive-dyes/reactive-red-120.html, acessado em 03/11/2013 